\title{
Measurement of Torsion from Multitemporal Images of the Eye Using Digital Signal Processing Techniques
}

\author{
J. ANTHONY PARKER, MEMBER, IEEE, ROBERT V. KENYON, MEMBER, IEEE, \\ AND LAWRENCE R. YOUNG, FELLOW, IEEE
}

\begin{abstract}
Two methods of measuring ocular torsion from digital images of the eyes were developed and tested. One method measures torsion from the translation of two landmarks using a rectilinear coordinate system. The second method measures torsion from the translation of two landmarks using a polar coordinate system. The center for the polar sampling is the center of the pupil. After thresholding and filtering the images, landmark translation is measured from the interpolated peak in the normalized cross correlation of the reference landmark with the image.

The standard deviation of the measurement error for the first method using artificially rotated well-framed $256 \times 256 \times 8$ single-eye images was $0.042^{\circ}$ in the absence of noise and $0.061^{\circ}$ for a noise-to-signal ratio of 0.1. The corresponding measurement accuracies for the radial sampling method were $0.019^{\circ}$ and $0.031^{\circ}$. The precision of the torsion measurement for high-quality experimental images was $0.132^{\circ}$. The landmark tracking method on the rectilinear grid can be used when the rotation is within a $\pm 5^{\circ}$ range. The measurement technique using the polar sampling can be used when there is a single point which is moderately well known.

Thus, digital signal processing techniques can be used to measure ocular torsion from images of the eye with a precision similar to the precision obtained by human photographic interpretation. The precision of the measurement does not appear to be limited by the precision of the digital processing technique.
\end{abstract}

\section{INTRODUCTION}

M EASUREMENT of ocular counterrolling-torsion of the eyes about the optic axis in response to head tilt-is valuable for understanding the physiology of the otolith organs because the ocular counterrolling response is one of the few relatively direct indications of otolith function [1]. There has been increased interest in otolith function with the advent of manned space flight since the otolith organs are believed to play an important role in motion sickness-a frequent problem in space travel [2], [3]. There is also interest in ocular torsion elicited from semicircular canal stimulation, and in the interaction between canal and otolith function.

While good systems for measurements of horizontal and vertical rotation of the globe have been available for many years [4], measurement of ocular torsion is more difficult to perform

Manuscript received July 29, 1983; revised January 18, 1984. This work was supported by the James Picker Foundation under Grant SA76-6, by the NASA Ames Research Center under Grant NAG2-88, and by the General Clinical Research Centers Program of the Division of Research Sources, National Institutes of Health under Grant RR-01302.

J. A. Parker is with the Division of Nuclear Medicine, Beth Israel Hospital, Boston, MA 02215, and the Department of Radiology, Harvard Medical School, Boston, MA.

R. V. Kenyon and L. R. Young are with the Man-Vehicle Laboratory, Department of Aeronautics and Astronautics, Massachusetts Institute of Technology, Cambridge, MA 02139. accurately and conveniently. Invasive methods of measuring ocular torsion involved attachment of a device or substance to the eye which can be easily tracked. Ink [5] or barium sulfide [6] have been applied to the eye, sutures have been placed in the subconjunctiva [7], and the cornea has been tatooed [8]. The advent of hard contact lenses provided a somewhat less invasive means of providing landmarks for tracking. However, to prevent the lenses from slipping, "suction caps" were designed with a chamber which could be evacuated so that the lens would stick into the cornea. Mirrors [9], coils [10], and magnets [11] have been attached to the suction caps to measure torsion. Use of suction systems is not always possible since the lenses must be custom fit and the evacuation of the chamber between the lens and cornea causes corneal deformation, increased ocular pressure, possible corneal abrasions, and necessitates the use of corneal anesthetics.

Ocular torsion has been measured noninvasively by tracking natural landmarks on the eye. The peripherally located conjunctival blood vessels are not firmly fixed to globe [12] so that the best results are obtained by using landmarks located within the iris. However, identification of iral landmarks is difficult due to the similarity of the ocular features about the axis of rotation. The most effective methods have used photographic images of the eye which are analyzed by hand [13][15]. Torsion is calculated from the motion of two or more of these landmarks.

This paper describes two similar noninvasive methods of measuring ocular torsion in man which use digital signal processing methods to analyze images of the eye. Torsion is calculated by tracking landmarks on the iris similar to the methods using human photographic interpretation. The signal processing methods are slightly more complex than those recently implemented in hardware by Hatamian [16], [17]. The major source of error with that implementation was due to errors in locating the pupil center. Our method has the advantage that it does not require accurate identification of the center of the pupil. This paper is divided into two sections-analysis of the effect of the digital signal processing methods on the accuracy of the torsion measurement, and determination of the overall accuracy of these methods on experimental data.

\section{Measurement Accuracy: Analysis of the Signal Processing Algorithm}

Single-eye $35 \mathrm{~mm}$ images of a blue-eyed (Fig. 1) subject performing ocular torsion, obtained from a previous experiment, were used to develop and verify image processing for ocular 


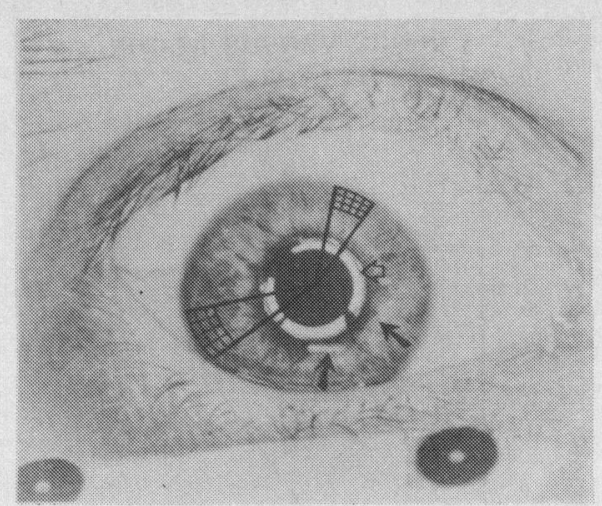

Fig. 1. Selection of landmarks. An image of a blue-eyed subject is shown with two landmark samples with the sector tracking method. The grid lines are an artistic representation of the digitization of the two sectors. The open arrow shows the light reflex from a circular flash. The closed arrows show reflections from the cornea.

torsion measurement. $8 \times 10$ glossy prints of the images were made. The images were then digitized with an Autokon laser image digitizer (ECRM, Bedford, MA) using $512 \times 512$ pixels (picture elements). Included within the image of the eye were two fiducial marks. The fiducial marks, which were attached to a bite bar, were used to indicate the position of the skull. A subregion of approximately $256 \times 256$ pixels covered the region of the iris and pupil.

The images were analyzed using a PDP $11 / 34$ computer system with 256 kbytes of memory and two Control Data Corporation 9766300 Mbyte disk drives. The GAMMA-11 software package (Digital Equipment Corporation) was used to select landmarks on the images and to display images on a VSVO1 video display. However, landmarks could be identified through terminal $\mathrm{I} / \mathrm{O}$ or light from input over the digitized eye image. Programming was done in the Ratfor language which was preprocessed to produce Fortran [18], [19]. The Fortran was compiled with the RT11 Fortran compiler. The algorithms for measurement of translation and rotation are given in the Appendix.

\section{Measurement of Landmark Translation}

Using a subimage from the iral detail, several experiments were performed to measure the accuracy of the translation measurement and to identify the effects of rotation, noise, and resolution on the accuracy of the measurement of translation. Measurement of landmark translation is important since it is used in both the landmark and the sector tracking algorithms. In order to study the image processing algorithms without biological or instrumentation effects, the accuracy of the algorithms was tested on mathematically rotated and translated images.

Accuracy of the Translation Measurement: Three $64 \times 64$ subimages of the iral detail were extracted from a $256 \times 256$ image of a blue-eyed subject which was well framed about the eye. These subimages were digitally translated by $0.1,0.2$, $0.3,0.4$, and 0.5 pixels. Square landmarks which were $3 \times 3$, $5 \times 5,9 \times 9,17 \times 17$, and $33 \times 33$ pixels were selected from the initial image. These landmarks were then tracked using the landmark tracking algorithm on the artificially translated images. Data were obtained for the three subimages, for the five different sized regions, and for the five translations. The
TABLE I

Accuracy of the Measurement of Translation. Measurement Error (Pixels)

\begin{tabular}{ccc}
\hline Translation & Mean & Standard Deviation \\
\hline 0.1 & -0.062 & 0.029 \\
0.2 & -0.088 & 0.042 \\
0.3 & -0.131 & 0.044 \\
0.4 & -0.092 & 0.030 \\
0.5 & -0.014 & 0.031 \\
\hline
\end{tabular}

TABLE II

Effect of Rotation on the Accuracy of Translation Measurement. Measurement Error (Pixels)

\begin{tabular}{|c|c|c|}
\hline Rotation & Mean & Standard Deviation \\
\hline 1 & -0.021 & 0.045 \\
\hline 2 & -0.029 & 0.052 \\
\hline 3 & -0.029 & 0.060 \\
\hline 5 & -0.043 & 0.088 \\
\hline 10 & -0.092 & 0.179 \\
\hline
\end{tabular}

errors in the measured translation as compared to the true translation were recorded as an indication of the accuracy of the measurement.

The means and the standard deviations of the errors in the translation measurement as a function of the translation are shown in Table I. There is a systematic underestimation of the translation for translations from 0.1 to 0.4 pixels with the largest underestimation for 0.3 pixels. The underestimation may be due to the interpolation function which was used to produce the images with subpixel translations. The interpolation function weighs the nearest pixel more heavily than would a linear interpolation [20].

The accuracy (standard deviation of the error) of the measurement of translation for the whole table was 0.052 pixels. This value is larger than the standard deviations at each of the offsets. Some of the variability in the data is simply due to the systematic error at each offset.

\section{Effect of Rotation on the Accuracy of Translation Measure-} ment: The subimages used in the translation experiment were digitally rotated by $1^{\circ}, 2^{\circ}, 3^{\circ}, 5^{\circ}$, and $10^{\circ}$. The center of rotation and the center point for polar sampling were at a different location. Again landmarks which were $3 \times 3,5 \times 5$, $9 \times 9,17 \times 17$, and $33 \times 33$ were selected on the initial image. These landmarks were tracked on the artificially rotated images and the errors in the tracking method were recorded.

The means and the standard deviations of the errors in the translation measurement as a function of the angle of rotation are shown in Table II. The accuracy (standard deviations of the error) is shown graphically in Fig. 2. Up to about a rotation of $5^{\circ}$, the accuracy of the measurement of translation is quite good. Above that angle, there is some decrease in the accuracy.

\section{Effect of Noise on the Accuracy of Translation Measurement:} In order to determine the effect of detector noise on the accuracy of the translation measurement, Poisson-distributed pseudorandom noise was added to the initial image and the translation experiment was repeated. A major component of 


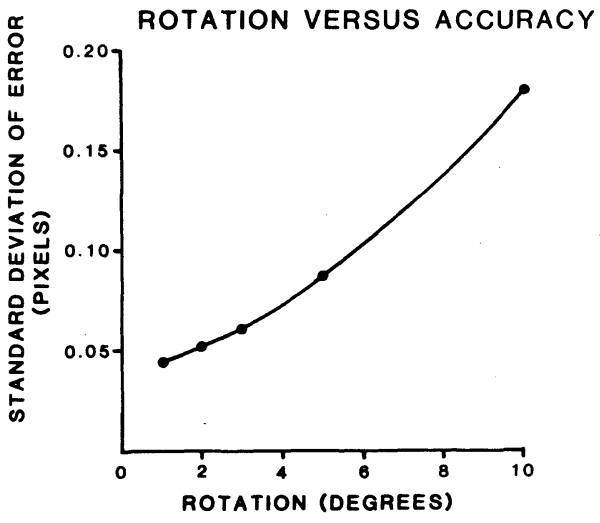

Fig. 2. Effect of rotation on the accuracy of the measurement of landmark translation. The accuracy (standard deviation of the error) of the measurement of translation is shown as a function of the relative rotation between the reference landmark and the test image for artificially rotated images.

the detector noise is often Poisson distributed. Again the errors in measurement as compared to the known translations were recorded.

Poisson-distributed pseudorandom noise was produced using samples from the Fortran pseudorandom noise generator. The RT11 Fortran pseudorandom noise generator produces noise uniformly distributed between 0 and 1 . When the mean $m$ was less than or equal to $10, n$ times $m$ samples were obtained from the uniform distribution. The number of samples less than $1 / n$ was taken as an approximation to the Poisson distribution. For this experiment $n$ was set equal to 10 (a true Poisson distribution is produced in the limit as $n$ goes to infinity). When the mean value was greater than 10 , the Poisson distribution was assumed to be approximately Gaussian. The Gaussian distribution was approximated by adding ten samples of the uniform distribution (multiple samples of any distribution approximate a Gaussian distribution by the central limit theorem).

The standard deviations of the errors in measuring landmark translation as a function of the amplitude of the noise added to the image are shown in Table III for the landmarks which could be successfully tracked. These data are shown graphically in Fig. 3. There is relatively little effect of noise on the accuracy of the translation measurement for noise-to-signal ratios less than 0.25 . There was a much more pronounced effect of the noise on the number of landmarks which could not be successfully tracked. Failures occurred when the noise overwhelmed the peak in the cross correlation. The failures occurred for the smaller landmark sizes. With no added noise and with a noise-to-signal amplitude ratio of 0.01 there were some failures for $3 \times 3$ sized landmarks, but no failures for large landmarks. For a noise-to-signal amplitude ratio of 0.10 , there were no failures for landmarks larger than $9 \times 9$, and for a noise-to-signal amplitude ratio of 0.25 , there were no failures for landmarks which were $17 \times 17$.

Effect of Resolution on the Accuracy of the Translation Measurement: In order to determine the effect of resolution on the accuracy of the translation measurement, the translation experiment was repeated after low-pass filtering the images. Two ideal low-pass filters of $\frac{1}{2}$ and $\frac{1}{4}$, the original sampling resolution, were used. The filtered images would correspond

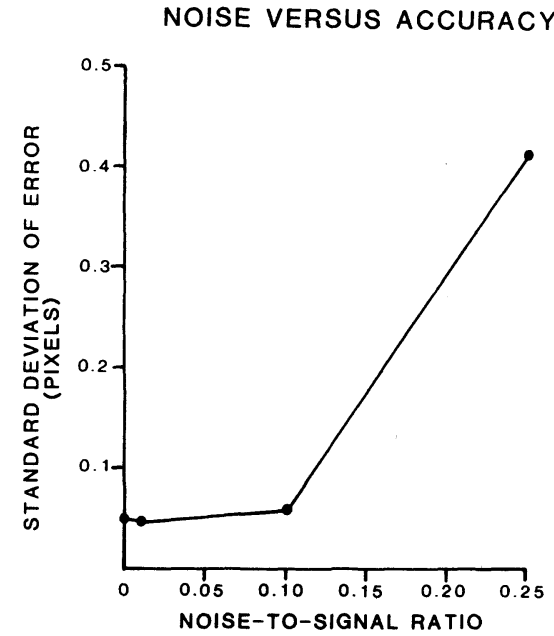

Fig. 3. Effect of noise on the precision of the measurement of landmark translation. The precision (standard deviation of the error) of the measurement of translation is shown as a function of the amount of noise added to the image.

TABLE III

Effect of Noise on the Accuracy of Translation Measurement. Measurement Error (Pixels) When Tracking Was Successful

Noise/Signal Standard Deviation

$\begin{array}{ll}0 & 0.047 \\ 0.01 & 0.046 \\ 0.10 & 0.057 \\ 0.25 & 0.414\end{array}$

TABLE IV

Effect of Resolution on the Accuracy Translation Measurement. Measurement Error (Pixels)

\begin{tabular}{cc}
\hline Dimension & Standard Deviation \\
\cline { 2 - 3 } $256 \times 256$ & 0.047 \\
$128 \times 128$ & 0.066 \\
$64 \times 64$ & 0.108 \\
\hline
\end{tabular}

to a well-framed eye image of $128 \times 128$ pixels and $64 \times 64$ pixels, respectively. Accuracies of the landmark tracking are given in terms of the original image dimensions to facilitate comparison.

The accuracy (standard deviation of the measurement error) of the measurement of translation as a function of image dimension is shown in Table IV. The same data are shown in Fig. 4. There is a decrease in the measurement accuracy as the dimension decreases. Much of the iral detail is lost at resolutions less than $64 \times 64$ pixels. Thus, there should probably be at least $64 \times 64$ pixels over the iris.

\section{Measurement of Image Rotation}

Algorithms: The two algorithms used for measurement of rotation are described in the Appendix. The landmark tracking algorithm measures the rotation by tracking two operator selected iral landmarks. From the distance between the landmarks and the translation of the landmarks, the rotation of the eye with respect to a reference image can be calculated. The second algorithm, the sector tracking algorithm, is similar to the landmark tracking algorithm except that the two landmarks are sampled on a polar coordinate system. The center 


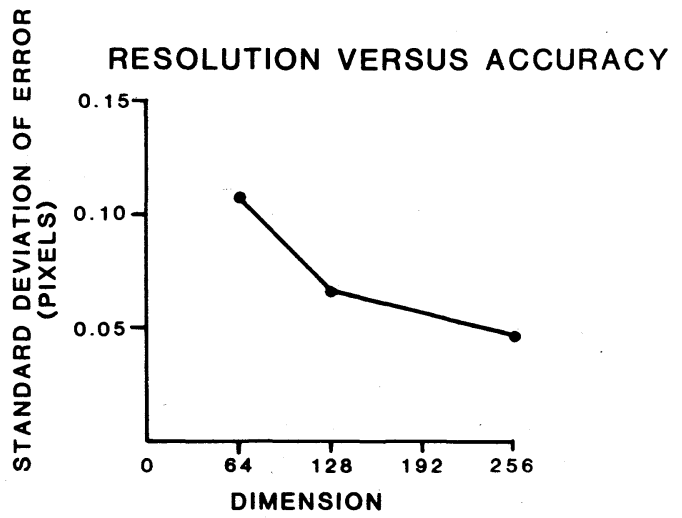

Fig. 4. Effect of resolution on the accuracy of the measurement of landmark translation. The accuracy (standard deviation of the error) of the measurement of translation is shown as a function of the resolution of the image. The dimension is the number of pixels in the horizontal and vertical directions for a well-framed image of a single eye.

of sampling for the polar coordinate system used in the sector tracking method can be any point which is fixed on the object which is rotating. The center of the pupil which is roughly the center of rotation has been used in the application of this technique to measurement of ocular counterrolling; however, any fixed point could be used.

Accuracy of Measurement on Artificially Rotated Images: In order to determine the accuracy of the two algorithms in measuring rotation, a $256 \times 256$ well-framed image of a blueeyed subject was digitally rotated by $1^{\circ}, 2^{\circ}, 3^{\circ}, 5^{\circ}$, and $10^{\circ}$. The landmark tracking and the sector tracking algorithms were applied to the image and the errors in the measurement of rotation were recorded. For both the landmark tracking and sector tracking algorithms, three different pairs of landmarks were used, and the experiment was repeated at a noise-to-signal ratio of 0.1 . Since the landmarks were about 100 pixels apart, there were about 2 pixels per degree.

The means and the standard deviations of the measurement error are shown in Table $\mathrm{V}$ for both the landmark tracking and the sector tracking algorithms for both no added noise and for zero indicating that there is no evidence of a systematic error in the calculation. The standard deviation of the error is quite small-about $\frac{1}{20}$ of a degree for the landmark tracking algorithm and less than $\frac{1}{30}$ of a degree for the sector tracking algorithm. For both algorithms the error is larger with added noise.

Effect of Center Location on Precision of the Sector Tracking Algorithms: The sector tracking algorithm depends upon identifying one point in both images which can be used as the center for the polar sampling. Since the center of the pupil is fairly readily identified, it has been used as the center point for the radial sampling. However, precisely locating the pupil center may sometimes be difficult; therefore, we examined the effect of the center point location on the accuracy of the rotation measurement. The sector tracking experiment was repeated with intentional center offsets on the initial image of 5 and 10 pixels. Three sectors $45^{\circ}$ apart were selected and the rotation measurement experiment was repeated.

Table VI shows the results of the experiment using a center for the radial sampling which was intentionally offset from the reference to the test image. There is an increase in the standard deviation of the measurement of rotation (a decrease in accuracy) with an increase in offset. However, the accuracy re-
TABLE V

Accuracy of the Measurement of Rotation. Measurement Error (Degrees)

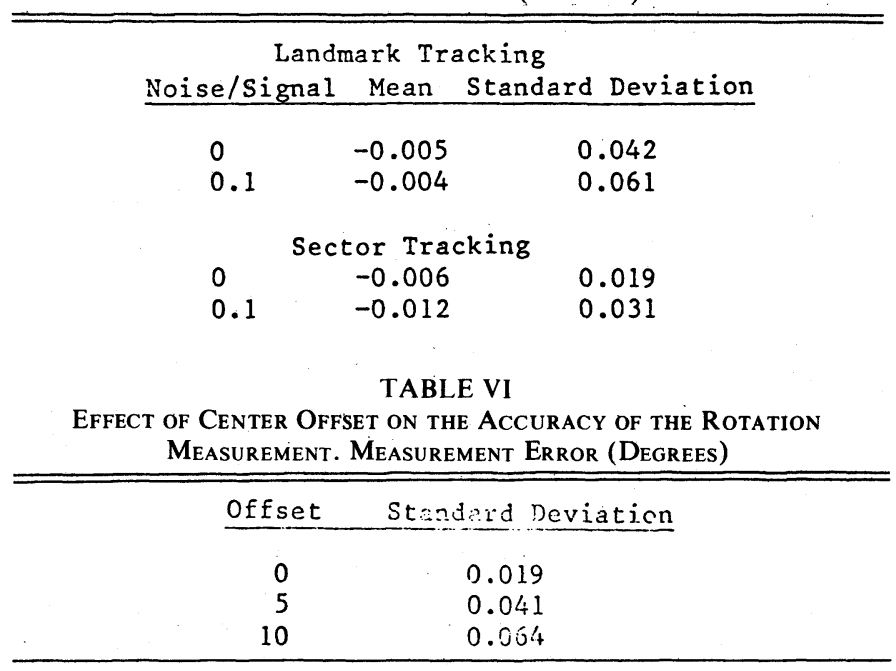

mains good, $0.064^{\circ}$, with a 10 pixel offset. Thus, the measurement of rotation with the sector tracking algorithm is relatively insensitive to moderate errors in the location of the center point.

\section{Precision of Measurement on EXPERIMENTAL IMAGES}

The results of the measurements on the artificially rotated image are best for defining the accuracy of the digital signal processing method; however, to evaluate physiologic and instrumentation effects, these methods were also tested on experimental images. Three sets of images were analyzed in order to determine the experimental precision. Two sets were from experiments on blue-eyed subjects and one set was from a brown-eyed subject. Although the blue-eyed subjects had much more iral detail, the brown-eyed subject did have some landmarks which could be identified by visual inspection. One set of data from one of the blue-eyed subjects was digitized with a high-quality laser image digitizer (Autokon, ECRM, Bedford, MA). The two other sets of data were digitized with a lower resolution video system (Matrox UFG-01, URGB-256).

For each set of images, one image was used as the reference image, and the other images were used as the test images. The rotation of the globe was calculated using several landmarks and several sectors for each of the test images. The square root of the mean-square error of the various measures was calculated as a measure of the precision of the rotation measurement.

Table VII shows the square root of the mean-square error for the measurement of rotation between the various images of the three experiments. These data show the precision of the rotation calculation for real images. For the best quality data the precision of the measurement was about $0.1^{\circ}$. This precision is considerably worse than the accuracy of measurement of the data for the digitally rotated images.

For the high-quality images, there was relatively little difficulty picking landmarks which would result in successful landmark tracking. For the lower quality images, several of the landmarks which were selected could not be tracked. Failures were readily identified by a large change in the distance between the landmarks on the reference and the test images. As 
RIGHT EYE

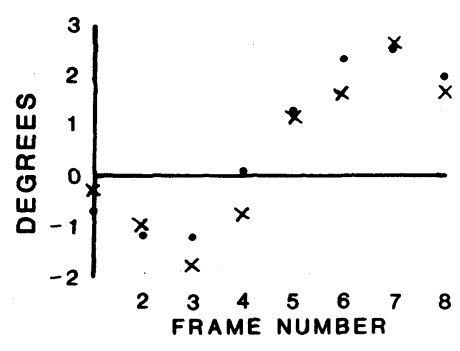

LEFT EYE

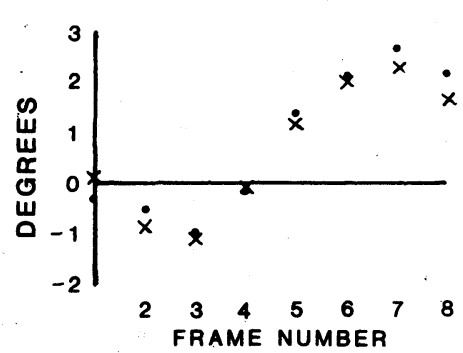

Fig. 5. Comparison of manual and automatic measurement of ocular torsion. Images from a sinusoidal acceleration experiment were analyzed by hand, crosses, and with the digital signal processing method, dots. The subject was accelerated laterally at $0.8 \mathrm{~g}$ at a frequency of $0.3 \mathrm{~Hz}$ on a specially constructed sled. The resolution over the area of the eye was $64 \times 64$ pixels.

TABLE VII

Precision of Rotation Measurement on Experimental Data. MEASURement ERror (Degrees)

\begin{tabular}{cc}
\hline Data Set & Exror \\
\hline Blue-eyed, high quality & 0.132 \\
Brown-eyed, good quality & 0.344 \\
Brown-eyed, low quality & 1.600 \\
\hline
\end{tabular}

a general rule, if the human photographic interpreter could not identify the landmark on successive images, the digital methods failed. Furthermore, certain types of artifacts affected the digital methods more than the analog methods. For example, if the landmark was overlayed by an image artifact such as a light reflex, the human could often identify the landmark while the digital method was much less successful.

Fig. 5 shows the application of the landmark tracking method to a sinusoidal acceleration experiment on a blue-eyed subject. The subject was accelerated at $0.8 \mathrm{~g}$ in the lateral direction at a frequency of $0.3 \mathrm{~Hz}$. Images of both eyes and a pair of fiducial marks were digitized with the laser digitizer. The fiducial marks were attached to a bite bar so that they indicate the position of the head. The fiducial marks were tracked with the same digital signal processing method and the torsional measurement was corrected for changes in the head position. The images were digitized using a $512 \times 512$ sampling matrix. This sampling density resulted in approximately $64 \times 64$ pixels over the eye.

The results of the digital method are compared to the human photographic interpretation in Fig. 5. Two-eye data are particularly demanding since without using very large sampling matrices, the resolution over the eye $(64 \times 64)$ results in considerable loss of the iral detail. Even with these difficulties, it was possible to track ocular torsion.

\section{Discussion}

Since both the landmark and sector tracking algorithms rely on the ability to measure landmark translation from a reference to a test image, factors affecting landmark tracking were examined in some detail. There are three data processing operations which can be used to improve the tracking-thresholding, selecting the landmark, and filtering. Thresholding the image so that the intensity values are spread out over the details of interest increases the energy of the landmarks with respect to the energy of the surrounding data. Therefore, the peak of the cross-correlation function increases with respect to the side lobes.

Selecting a prominent, distinctive landmark without artifacts such as light reflex is important for the tracking. We left the selection of the location of the landmark to the operator. Since the landmarks only need to be selected once on the reference image, the work of selecting the landmarks is not great. However, it is possible that an algorithm could be developed which would select the landmarks automatically.

Filtering is used to select the frequency components which are most prominent in the landmark. Iral detail has a considerable amount of high-frequency content as compared to the rest of a typical eye image. Therefore, amplifying the high frequencies by filtering the image tends to increase the power in the iral detail as compared to the background. Filtering, as does thresholding, increases the peak as compared to the sidelobes. In addition, since the typical eye images have less power at high frequencies, amplifying the high frequencies tends to narrow the peak of the cross-correlation function.

The precision of the measurement on experimental images was found to be good. For the high-quality images the precision of the rotation measurement was $0.13^{\circ}$. This precision is comparable to the precision of the best methods of measuring ocular torsion from photographs [13], [15], [21]. The accuracy of measuring torsion on artificially rotated images is, however, considerably better, about $0.03^{\circ}$. One explanation of why the precision of the measurement of the experimental images is less than the accuracy of measuring artificially rotated images is that there may be changes in the relation of the positions of the landmarks. The iral landmarks are due to the muscles in the iris. These muscles change position when the pupil dilates and constricts. There is always some small amplitude dilitation and constriction of the pupil, and there may be movement of these landmarks from image to image independent of dilitation or constriction. This explanation would also account for the similarity between the precision of the human photographic interpretation method of measuring rotation and the digital signal processing method of measuring rotation.

Many experiments require a range for the torsion measurement of about $\pm 10^{\circ}$. Since the landmark tracking algorithm begins to have difficulty above $\pm 5^{\circ}$, application of this algorithm would require some modifications. (For example, tracking could be done with several sets of reference landmarks which were rotated by $5^{\circ}$ increments.) The sector tracking algorithm overcomes these difficulties, but it requires that one point in the sequence of images be known with a modest (5 pixel) accuracy. Depending upon the experiment and the image processing capabilities, one algorithm may be preferred over the other. 
When comparing the digital methods of tracking iral landmarks to the human photographic interpretation method of tracking iral landmarks, the digital methods seem to be at least as accurate when they can be successfully applied. The digital methods do, however, have more difficulty with identification of landmarks, especially when the landmarks are obscured by artifacts. Human photographic interpretation is less troubled by overlying artifacts such as light reflections since the human interpreter is able to use much more contextual information to separate the landmark from the overlying artifact.

Since the purpose of this investigation was to develop algorithms for tracking torsion, the image processing was carried out on a relatively slow general purpose computer. A more powerful computer or an array processor would be required for practical implementation of these algorithms. Image processing systems of the required power are becoming available at moderate cost. Real-time implementation of image analysis algorithms of slightly less complexity has been reported by Hatamian [16], [17] using special purpose hardware. Thus, practical application of these digital signal processing methods to measurement of ocular torsion should be possible in the near future.

\section{APPENDIX}

\section{Digital Signal Processing Algorithms}

More details on the digital signal processing algorithms can be found in [22]. In remotely sensed imagery, such as LANDSAT imagery, it is common to measure both the translation and rotation of images as a first step in the registration of images which have been acquired with different sensors or which have been acquired at different times [23]-[25]. The rotation measurement is generally performed by measuring translations of two or more landmarks. Measuring the rotation from the translation of two landmarks assumes that the rotated landmarks can be identified. Since the rotation of the images is often of relatively small angle, tracking the landmarks is relatively easy.

In this paper, two slightly different rotation measurement algorithms were used. The first algorithm, which will be called "landmark tracking," is similar to the rotation measurement algorithms used for remotely sensed images. Rotation is calculated from the distance between the landmarks and the translation of the landmarks. The second algorithm, which will be called "sector tracking," performs the same operation of tracking two landmarks, but the tracking is performed on a polar coordinate system instead of on a rectilinear coordinate system. Use of the polar coordinate system improves the performance on images with a large relative rotation.

\section{Methods Common to Both Algorithms}

\section{Landmark Selection}

Landmarks with a considerable amount of detail were selected from the images by the computer operator. Landmarks which had overlying artifacts such as light reflexes were avoided (Fig. 1). Generally, the best performance was obtained from landmarks which were most distinctive to the operator. Two landmarks were selected on opposite sides of the pupils.

Landmark selection amounts to windowing or masking the reference image. The best window for use with measurement of translation has been studied by Mostafavi [26], [27]. For a landmark with an autocorrelation function which is a delta function, a heuristic rule is that the window should be large enough so that the rotation between the reference and test images results in one pixel offset across the size of the window.

\section{Interpolation}

To measure the accuracy of the technique for measuring translation, it was necessary to digitally translate the images by subpixel amounts. To measure the accuracy of the rotation measurement, it was necessary to digitally rotate the images. Both rotation and subpixel translation require that the image data be resampled from the original coordinate points to a new set of coordinate points. A cubic spline interpolating function defined over the four nearest points was used in this paper [20], [28]

$$
\begin{aligned}
& \qquad \begin{array}{l}
\frac{3}{2} x-\frac{5}{2} x+1 \\
-\frac{1}{2} x+\frac{5}{2} x-4 x+2
\end{array} \\
& \text { Filtering }
\end{aligned}
$$

The images were digitally filtered prior to measurement of translation with a filter which increased the high frequencies in order to produce a sharper peak in the autocorrelation function [22]. The filter function which was used was a second difference of a Gaussian with a zero crossing at 1 pixel distance. This function removes the dc component of the image and enhances the higher frequency components. It attenuates the highest frequency components, those components which are present only in the diagonal directions.

\section{Measurement of Landmark Translation}

Measurement of a translation of an object from two images of the object is often performed by comparing the two images at different offsets using a similarity measure. The similarity measure is compared at different relative translations and the true translation is taken to be the point at which the two images are most similar. The most commonly used similarity detection algorithm is the normalized cross correlation.

Cross correlation is a well-known operation-the integral of the product of one function times the conjugate of an offset version of the second function. The cross correlation of the landmark $f_{1}$, with the test image $f_{2}$, is given by

$$
R_{f_{1} f_{2}}\left(i^{\prime}, j^{\prime}\right)=\operatorname{sum}_{i, j}\left(f_{1}(i, j) f_{2}^{*}\left(i-i^{\prime}, j-j^{\prime}\right)\right)
$$

where the star indicates the conjugation operation. $R_{f_{1} f_{2}}$ is defined only for those points where the landmark and the test image completely overlap.

The magnitude of the unnormalized cross correlation is not a good measure of similarity between images because it is dependent upon the energy of those portions of the image which are included in the cross correlation [23]. The energy of an image is defined in the usual fashion

$$
e=\operatorname{sum}_{i, j}\left(f(i, j) f^{*}(i, j)\right) .
$$

If the portion of the image from which the subimage is taken does not have the greatest energy in the image, then the noise in the cross-correlation function at other positions may produce a greater value of $R_{f_{1} f_{2}}$ than the value at the true matched position. 
In order to overcome this problem, the cross-correlation function is normalized for the energy

$$
R_{f_{1} f_{2}}^{\prime}\left(i^{\prime}, j^{\prime}\right)=R_{f_{1} f_{2}}\left(i^{\prime}, j^{\prime}\right) /\left(e_{f_{1}} e_{f_{2}}\right)^{1 / 2} \text {. }
$$

The normalized cross correlation will have a value from -1 to 1 , where a value of 1 implies the images are identical up to a scale factor. The normalized cross correlation has been used for image registration by a large number of authors (for example, [23] , [29]-[32]).

The number of operations to calculate the normalized cross correlation increases on the order of the square of the number of pixels. In order to improve this performance, the cross-correlation operation is often implemented with the fast Fourier transform (FFT) algorithm [29] .

$$
R_{f_{1} f_{2}}=F T^{-1}\left(F T\left(f_{1}\right) F T^{*}\left(f_{2}\right)\right)
$$

where $F T$ is the Fourier transform operation and $F T^{-1}$ is the inverse Fourier transform operation. An efficient method of calculating the energy for the different offsets is to correlate a mask of the landmark $m$ with the square of the magnitude of the image. The mask $m$ has a value equal to one inside the landmark and zero outside the landmark.

$$
e=F T^{-1}\left(F T(m) F T^{*}\left(f^{2}\right)\right) \text {. }
$$

The Fourier transform implemented with the Fourier transform algorithm will increase with image size only as $N \log N$ (where $N$ is the number of pixels in the image).

\section{Rotation Calculation}

The data needed to calculate the rotation of the two images are the translation of two landmarks (1 and 2) from the reference image $a$ to the test image $b$ and the distance between the centers of the two landmarks. If point $a$ is translated to the origin, then point $b$ rotates about $a$. This rotation can be expressed in terms of the new coordinates

$$
\begin{aligned}
& (x 1, y 1)=(x 1 b-x 1 a, y 1 b-y 1 a) \\
& (x 2, y 2)=(x 2 b-x 2 a, y 2 b-y 2 a) .
\end{aligned}
$$

The angle of rotation is given by

$$
\text { theta }=\arctan \left(x_{1} * y_{2}-x_{2} * y_{1}\right) /\left(x_{1} * x 2+y 1 * y_{2}\right) .
$$

\section{Rotation Measurement Algorithms}

\section{Landmark Tracking}

The iral detail in the eye images can often be made more prominent by a nonlinear thresholding operation where all values above the upper threshold are set to the maximum. Each of the landmarks is extracted from the reference image into a subpicture which is at least large enough to hold it. The subpicture must have dimensions which are a power of 2 greater than or equal to 64. A subpicture which is located in the same position is then extracted from the test image. Because the use of the subpictures limits the cross-correlation search area, processing time is reduced. Limiting the search assumes that the images are roughly aligned; however, since the eye is usually near the center of the image, this restriction is not very severe.

A mask image of ones and zeros corresponding to the landmark is produced. The center of mass of this image is used as the location of the landmark. The region of interest from the reference image is then cross correlated with the subpicture from the test image using a normalized cross correlation. The peak of the cross-correlation function is used as a measure of the translation of the landmark between the two images. The peak is calculated with a parabolic interpolation so that subpixel translations can be measured. The parabolic interpolation was chosen as the simplest function which would provide subpixel values for the peak.

\section{Sector Tracking}

The operator needs to identify the approximate centers of the pupils and the landmarks which are to be tracked. Two new pictures are created by polar sampling of the reference and the test image from the center of the pupil. The horizontal dimension of the output file and the starting and ending angle determine the angular resolution. The vertical dimension of the output file and the starting and ending radius determine the radial resolution. The resolution in the angular direction of the inner arcs is greater than the outer arcs. The resampling is typically done at the resolution of the raw images at the center of the sampled region.

These radially sampled images are then two dimensionally cross correlated. The translation between these two matrices in the horizontal direction corresponds to the angular direction and provides a measure of the rotation of the sampled region between the two images. The translation between these matrices in the vertical direction corresponds to the radial direction and is a measure of the accuracy with which the center has been calculated.

The cross correlation of the two images is dependent on the accuracy of the location of the pupil center in the radial direction. If the two regions are badly misaligned with respect to the radial direction, the samples of the same area will be sampled at different resolutions and the arcs will have a different curvature. Thus, the distance over which similar portions of the two images can be made to line up will be reduced. Errors in the pupil center corresponding to the tangential direction will also distort the arcs so that they will correspond over a shorter distance. If the translation between reference and test image were not factored out (see below), errors in the tangent direction would translate directly into error in the measurement of rotation.

Errors in locating the center of rotation have been a major problem with implementation of the sector tracking method [16], [17]. In order to decrease the sensitivity to the center position, the positions of the two landmarks were calculated from the average radius and the average angle of the regions. With the positions of the two resampled regions and the offsets from the reference to the test image of these region, the same calculation which is used above in the landmark tracking method was used to calculate the rotation.

The advantages of using this calculation are that the errors more nearly cancel and that the angle between the two regions need not be $180^{\circ}$. Furthermore, this method is relatively insensitive to the location of the center of rotation as long as the center of rotation is the same in the reference and the test images. With this type of calculation, the sector tracking method becomes quite similar to the landmark tracking method. The major advantage of the sector tracking method over the land- 
mark tracking method is that its performance does not decrease with increasing rotation angle.

\section{REFERENCES}

[1] B. K. Lichtenberg, L. R. Young, and A. P. Arrott, "Human ocular counterrolling induced by varying linear accelerations," Exp. Brain Res., vol. 48, pp. 127-136, 1982.

[2] C. M. Oman, "A heuristic mathematical model for the dynamics of sensory conflict and moíion sickness," Act. Oto-Laryngologica, Suppl. 392, 1982.

[3] - "Space motion sickness and vestibular experiments in spacelab," SAE Tech. Paper Series 820833, 1982.

[4] L. R. Young and D. Sheena, "Survey of eye movement recording methods," Behav. Res. Method Instrum., vol. 7, pp. 397-429, 1975.

[5] J.P.C. Southall, Ed., Helmholtz's Treatise on Physiological Optics (Transl. from Third German Edition Vol. III. The Perceptions of Vision), Menasha, WI: The Optical Society of America, 1925.

[6] C. E. Benjamins, "L'examen de la fonction otolithique par la mesure de la contre-rotation des yeux (gegenrollung)," Rev. Laryngol. Otol. Rhinol. (Bord), vol. 47, pp. 111-118, 1926.

[7] A. Graybiel and R. C. Woellner, "A new and objective method for measuring ocular torsion," Amer. J. Ophthalmol., vol. 47, pp. 349-357, 1959.

[8] C. E. Benjamins, "Contribution a la connaissance des reflexes tonique des muscles de l'oeil," Archiv Neerlandaises Physiologie Homme Animaux, vol. 2, pp. 536-551, 1918 .

[9] D. H. Fender, "Torsional motions of the eyeball," Brit. J. Ophthalmol., vol. 39, pp. 65-72, 1955.

[10] D. A. Robinson, "A method of measuring eye movement using a scleral search coil in a magnetic field," IEEE Trans. Biomed. Eng., vol. BME-10, pp. 137-145, 1963

[11] Y. Y. Zeevi and J. Iah-Shalom, "Measurement of eye movement with a ferromagnetic contact ring," IEEE Trans. Biomed. Eng., vol. BME-29, pp. 511-522, 1982.

[12] P. A. Merton, "Compensatory rolling movements of the eye," J. Physiol., vol. 132, pp. 25P-27P, 1956.

[13] E. F. Miller and A. Graybiel, "A comparison of ocular counterrolling movements between normal persons and deaf subjects with bilateral labyrinthe defects," Ann. Otology, Rhinol. Laryngol., vol. 72 , pp. 885-893, 1972.

[14] S. G. Diamond, C. H. Markham, N. E. Simpson, and I. S. Curthoys, "Binocular counterrolling in humans during dynamic rotation," Acta Otolaryngol., vol. 87, pp. 490-498, 1979.

[15] R. Balliet and K. Nakayama, "Training of voluntary torsion," Invest. Ophthalmol. Visual Sci., vol. 4, pp. 303-314, 1978.

[16] M. Hatamian, "An image analysis algorithm for real time measurement on $X, Y$ and torsional eye movements-Theory and implementation," Ph.D. dissertation, Univ. Michigan, Ann Arbor, MI, 1982.

[17] M. Hatamian and D. J. Anderson, "Design considerations for a real-time ocular counterroll instrument," IEEE Trans. Biomed. Eng., vol. BME-30, pp. 278-288, 1983.

[18] B.W. Kernighan and P. J. Plauger, Software Tools. Reađing, MA: Addison-Wesley, 1976.

[19] R. G. Beckmann, M. S. Sasaki, H. D. Royal, J. A. Parker, and G. M. Kolodny, "Incorporation of a RATFOR preprocessor into a nuclear medicine computer system," in Digital Imaging: Clinical Advances in Nuclear Medicine. New York: Society of Nuclear Medicine, 1982 , pp. $165-172$.

[20] J. A. Parker, R. V. Kenyon, and D. E. Troxel, "Comparison of interpolating methods for image resampling," IEEE Trans. Med. Imaging, vol. MI-2, pp. 31-39, 1983.

[21] L. R. Young, B. K. Lichtenberg, A. P. Arrott, T. A. Crites, C. M. Oman, and E. R. Edelman, "Ocular torsion on earth and in weightlessness," Ann. N.Y. Acad. Sci., vol. 374, pp. 80-92, 1981.

[22] J. A. Parker, "Measurement of torsion from aulti-temporal images of the eye using digital signal processing techniques," Ph.D. dissertation, Mass. Inst. Technol., Cambridge, MA, 1983.

[23] R. Bernstein and H. Silverman, "Digital techniques for earth resource image data processing," in Proc. Amer. Inst. Aeronautics Astronautics, 1971, vol. C21, pp. 1-1-1-14

[24] P. van Wie and M. Stein, "A landsat digital image rectification system," IEEE Trans. Geosci. Electron., vol. GE-15, pp. 130$137,1977$.
M. J. McDonnell, "Digital image rectification and enhancement on a minicomputer: Analysis of results," in Proc. 12th Int. Symp. Remote Sensing Environ., vol. III, 1978, pp. 1687-1695.

[26] H. Mostafavi, "Optimal window functions for image correlation in the presence of geometric distortion," IEEE Trans. Acoust., Speech, Signal Processing, vol. ASSP-27, pp. 163-169, 1979.

[27] H. Mostalavi, T. L. Steding, F. W. Smith, and R. S. Poulsen, “Optimum windows for image registration," IEEE Trans. Aerosp. Electron. Syst., vol. AES-17, pp. 101-110, 1981.

[28] R. G. Keys, "Cubic convolution interpolation for digital image processing," IEEE Trans. Acoust., Speech, Signal Processing, vol. ASSP-29, pp. 1153-1160, 1981.

[29] P. E. Anuta, "Spatial registration of multispectral and multitemporal digital imagery using fast Fourier transform techniques," IEEE Trans. Geosci. Electron., vol. GE-8, pp. 353-368, 1970.

[30] W. K. Pratt, "Correlation techniques of image registration," IEEE Trans. Aerosp. Electron. Syst., vol. AES-10, pp. 353-358, 1974.

[31] J. J. Pearson, D. C. Hines, S. Golosman, and C. D. Kuglin, "Videorate image correlation processor," Soc. Phot.-Opt. Instrum. Eng., vol. 119 , pp. 197-205, 1977.

[32] M. Singh, W. Frei, T. Shibata, C. Huth, and N. E. Telfer, "A digital technique for accurate change detection in nuclear medical images-With application to myocardial perfusion studies using Thallium-201," IEEE Trans. Nucl. Sci., vol. NS-26, pp. 565-575, 1979.

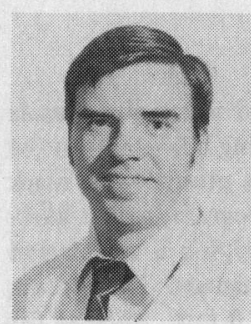

J. Anthony Parker (M'79) received the M.D. degree from Washington University, St. Louis, MO, in 1972, and the Ph.D. degree from the Massachusetts Institute of Technology, Cambridge, in 1983.

At present he is an Assistant Radiologist at the Beth Israel Hospital and an Assistant Professor of Radiology at Harvard Medical School, Boston, MA.

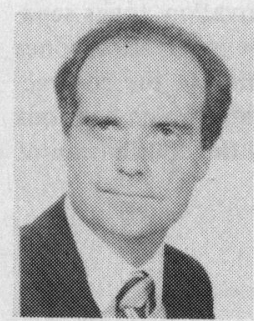

Robert V. Kenyon (S'71-S'78-M'78) received the B.S. degree from the University of Rhode Island, Kingston, in 1970, the M.S. degree in bioengineering from the University of Illinois, Urbana, in 1972, and the Ph.D. degree in physiological optics from the University of California in 1978.

Currently he is an Assistant Professor of Aeronautics and Astronautics at the Massachusetts Institute of Technology, Cambridge, working in the Man-Vehicle Laboratory. His research interests include sensory-motor adaptation, visuomotor control, instrumentation, flight simulation, and digital signal processing.

Dr. Kenyon is a member of Sigma Xi and the Biomedical Engineering Society.

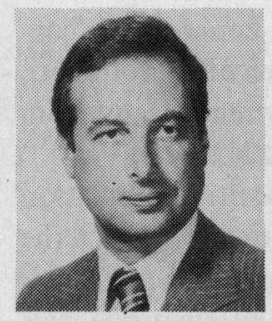

Lawrence R. Young (S'61-M'63-SM'75-F'79) received the A.B. degree from Amherst College, Amherst, MA, in 1957, the Certificat in applied mathematics from the Sorbonne, Paris, in 1958, the S.B. and S.M. degrees in electrical engineering, and the Sc.D. degree in instrumentation from the Massachusetts Institute of Technology, Cambridge, in 1962.

Currently he is a Professor of Aeronautics and Astronautics at M.I.T. and the Director of the Man-Vehicle Laboratory which does research on the vestibular and visual systems, visual-vestibular interaction, flight simulation, space motion sickness, manual control, and displays. His applications to the aerospace medical field have been in instrumentation (eye movement measurement) and basic and applied research in the field of vestibular function. His psychophysical work on semicircular canal and otolith function led to models which are applied in flight simulator motion control and are being extended to include visually induced motion effects. He is also recognized for his leadership in the aerospace applications of manual control theory, especially for his work on adap- 
tive manual control. His work on the vestibular system has led to an experiment on vestibular adaptation to weightlessness carried out on Spacelab 1 and several later shuttle missions. An avid skier, he has been investigating skiing injuries over the past several years. These investigations have ranged from purely statistical studies of reported injuries and the conditions contributing to them to measurements of how binding release characteristics are affected by weather and snow conditions. He is currently Chairman of the ASTM (American Standards of Testing and Materials) Subcommittee on Ski Injury Statistics.
Dr. Young is active on several professional and government committees, including the Air Force Scientific Advisory Board, the Space Medicine and Biology Committee of the National Academy of Science, and he was Chairman of the National Academy of Sciences Vestibular Panel of the Summer Study on Life Sciences in Space in 1977. He is also a member of the Barany Society for Vestibular Research and the Editorial Board of Neuroscience. He was President of the Biomedical Engineering Society during 1979 and was elected to the National Academy of Engineering in 1980. He is a Fellow in the Explorer's Club.

\title{
The Position of Innervation Zones in the Biceps Brachii Investigated by Surface Electromyography
}

\author{
TADASHI MASUDA, HISAO MIYANO, AND TSUGUTAKE SADOYAMA
}

\begin{abstract}
A linear surface electrode array placed along the muscle fibers detects motor unit action potentials propagating bilaterally to the tendons. The location of the propagation source is presumed to mark an innervation zone. We developed a computer program, which automatically determined the potential source by applying a correlation calculation and a linear regression to the recorded signals. The spacing between the contacts in the electrode array was $5.0 \mathrm{~mm}$, whereas for some recordings the histogram indicating the position of estimated source had a sharp peak concentrated in a $1.0 \mathrm{~mm}$ area. In the biceps brachii some subjects were found to have two innervation zones separated by $10-20 \mathrm{~mm}$. The peaks corresponding to the innervation zones differentially changed their histogram scores according to the contraction force. We also constructed an electrode assembly, which had four columns of the linear electrode arrays, and clarified the distribution of the innervation zones in the biceps brachii.
\end{abstract}

\section{INTRODUCTION}

$\mathrm{A}^{\mathrm{N}}$ $\mathrm{N}$ electromyogram (EMG) is a record of motor unit action potentials (MUAP's) which, originating from innervation zones, spread over the muscle bilaterally along the muscle fibers. An innervation zone is a cluster of myoneural junctions, or motor endplates, on muscle fibers and confined to a region of several millimeters under normal conditions. Most muscles have a small number of innervation zones which contain myoneural junctions for a large number of motor units. An individual MUAP is expected to spread symmetrically from the innervation zone to both ends of the muscle fibers.

In the EMG measurements, the electrode position in relation to innervation zones is important and is known to affect the characteristics of detected signals. For example, when myoelectric signals are derived bipolarly from an electrode pair placed on the skin surface, the recorded signals have different frequency distributions depending on the electrode position. If the electrodes are placed on the same side by avoiding the

Manuscript received September 20, 1983; revised June 29, 1984.

The authors are with the Industrial Products Research Institute, Tsukuba-gun, Japan, 305. innervation zones, they detect potentials propagating in a given direction with a given velocity, that is, the muscle fiber conduction velocity. Then, the detected signals are attenuated by a filter, which results from the electrode configuration and has nulls at frequencies determined by the electrode spacing and the conduction velocity [1]. However, if the innervation zone is positioned between the two contacts of bipolar electrode, MUAP's propagate in opposite directions under the contacts. In this case, the effective electrode spacing $\left(d^{\prime}\right)$, which determines the characteristics of the filter, decreases from the actual spacing $(d)$ to $d^{\prime}=d-2 r$, where $r$ is the distance between the innervation zone and the nearer one of the contacts [2]. In the extreme case, if the innervation zone confined to a small region is located just at the middle length of the electrode pair, each contact receives at any instant identical potential values because of the symmetry in the potential propagation and the effective electrode spacing $d^{\prime}$ becomes zero, because $2 r=d$. Consequently, nothing may be detected as a myoelectric signal. In fact, from the middle length of the biceps brachii Masuda et al. [3] have recorded a signal which had an extremely smaller amplitude (about one-fourth in the peak-to-peak amplitude) compared to the signals simultaneously obtained from other recording sites. However, most investigators in EMG studies, including the spectral analysis studies, have paid little attention to the position of the innervation zones.

Also, in the measurement of muscle fiber conduction velocity, the electrode position is crucial for the accurate and reliable recording of myoelectric signals. Several investigators have measured the conduction velocity using surface electrodes [1], [4]-[6]. However, they determined the electrode position based on an anatomical knowledge or on the motor point location found by the electrical stimulation technique [6]. General anatomical data do not describe the difference in individual subjects and, moreover, a relative position of the muscle and the skin may change depending on the bending angle of 